\title{
Consecuencias del desempleo en la salud mental de una muestra de jóvenes angoleños
}

\section{Consequences of unemployment on the mental health of a sample of Angolan youth}

\author{
José Manuel Tomás*, Melchor Gutiérrez ${ }^{* *}$ e Irene Fernández ${ }^{*}$ \\ *Departamento de Metodología de las Ciencias del Comportamiento, Universidad de Valencia, España

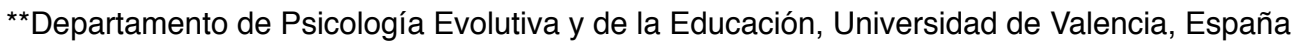

\begin{abstract}
Resumen
Entre los efectos del desempleo en la salud mental, destacan problemas de confianza, depresión y pérdida de recursos psicosociales. El objetivo es investigar las variables atenuantes del estrés sobre la salud mental en desempleados jóvenes angoleños mediante un modelo estructural con variables latentes. Se probaron los efectos del apoyo social, la resiliencia y las estrategias de afrontamiento sobre la salud mental, controlando por los efectos de ser económicamente suficiente. Para poner a prueba este modelo se ha contado con una muestra de 283 angoleños con edades comprendidas entre 18 y 30 años. El modelo se estimó en EQS 5.7. Los resultados indican un ajuste adecuado del modelo. Estos resultados apuntan a que apoyo social, resiliencia y estrategias de afrontamiento tienen un efecto atenuante sobre las consecuencias negativas del desempleo en la salud mental. Entre las implicaciones derivadas de este estudio destaca la necesidad de potenciar el apoyo de los padres, así como la necesidad de las instituciones sociales de encontrar modos de promover la búsqueda activa de empleo. Se discuten también los resultados obtenidos a la luz de la evidencia anterior y se sugieren las limitaciones derivadas del carácter transversal y de la muestra incidental del estudio.
\end{abstract}

\begin{abstract}
Commonly found effects of unemployment on mental health include a loss of confidence, a higher incidence of depression and a loss of psychosocial resources. Objective: To test the effect of strain-mitigating variables on mental health in a sample of unemployed Angolan youth using a structural model with latent variables. Effects of social support (from parents, friends, and others), resilience, and coping strategies on mental health were tested, while controlling for the effects of being economically self-sufficient. The model was tested on a sample of 283 Angolan youth (age range, 18--30 years). The structural model was estimated using EQS 5.7. The model had an adequate fit to the data. The results suggest that social support, resilience, and coping strategies have an alleviating effect on the negative consequences of unemployment on mental health. The results indicate the need to foster parental support and the need for social institutions to encourage the active search for employment. We discuss the results in the light of previous evidence and highlight the limitations related to the cross-sectional nature of the study and incidental sampling.
\end{abstract}

Key words: perceived social support, resilience, coping, mental health, Angola.

Palabras clave: apoyo social percibido, resiliencia, afrontamiento, salud mental, Angola.

Cómo citar este artículo: Tomás, J. M., Gutiérrez, M., y Fernández, I. (2017). Consecuencias del desempleo en la salud mental de una muestra de jóvenes angoleños. Escritos de Psicología, 10, 58-68.

Correspondencia: Profesor J. M. Tomás, Departamento de Metodología de las Ciencias del Comportamiento, Facultad de Psicología, Av. Blasco Ibáñez, 21, Valencia 46020. E-mail: tomasjm@uv.es. E-mail del coautor Melchor Gutiérrez: melchor. gutierrez@uv.es. E-mail de la coautora Irene Fernández: hyrenep@gmail.com. 


\section{Introducción}

El desempleo ha demostrado tener un fuerte impacto en la vida personal así como en el bienestar de los individuos que lo sufren, especialmente si éste es sostenido en el tiempo (Lorenzini y Giugni, 2011). Numerosos autores recalcan los efectos de la pérdida de trabajo en la salud mental (Björklund y Eriksson, 1998; McKee-Ryan, Song, Wanberg y Kinicki, 2005; Ślebarska, Moser y Gunnesch-Luca, 2009; Warr, Jackson y Banks, 1988). En el estudio meta-analítico realizado por McKee-Ryan et al. (2005), encontraron que el deterioro de salud mental, como consecuencia de la pérdida de trabajo, era la más comúnmente incluida en la literatura, y los resultados obtenidos en dicho meta-análisis sugieren un efecto negativo general del desempleo sobre la salud mental. También se señalan ciertas consecuencias negativas de carácter físico y fisiológico (Kinicki, Prussia y McKee-Ryan, 2000; Prussia, Fugate y Kinicki, 2001). Además, en un meta-análisis llevado a cabo por Jin, Shah y Svoboda (1995), se encontró relación entre el desempleo y mayor riesgo de enfermedades tanto mentales como físicas, además de un uso más elevado de los servicios sanitarios. Los efectos del desempleo en la salud mental tienen una mayor incidencia en los jóvenes (Bjarnason, y Sigurdardottir, 2003; Feather y O’Brien, 1986; Winefield, 1997). Entre las consecuencias psicológicas derivadas de la pérdida de empleo, se destacan los problemas de confianza (Amundson, 1994; Amundson, y Borgen, 1987), mayores niveles de depresión o disforia (Axelsson, y Ejlertsson, 2002; Bjarnason, y Sigurdardottir, 2003) y pérdida de recursos psicosociales (Kroll y Lampert, 2011). Por último, existe controversia entre la dirección causal de la relación entre la salud mental y el desempleo, ya que, conceptualmente, se podría plantear que el desempleo ejerce un efecto negativo en la salud mental, así como que una salud mental pobre conlleve la pérdida del trabajo, aunque la mayor parte de evidencia apunta a mayor probabilidad de una dirección en el sentido de que la pérdida de trabajo deteriora la salud mental, especialmente en sociedades con altas tasas de desempleo (Bjarnason y Sigurdardottir, 2003). La evidencia empírica parece indicar una relación unidireccional con el desempleo (Bjarnason y Sigurdardottir, 2003) teniendo una serie de consecuencias psicológicas. Esto va en línea con lo hallado por Jin et al. (1995), quienes reportan que en su revisión de estudios longitudinales, problemas significativos de salud tenían lugar tras la pérdida de empleo, mientras que no se tienen datos de ocurrencias de la situación contraria, esto es, un patrón de ocurrencia de problemas de salud antes de que se dé la situación de desempleo.

El apoyo social se puede entender como un recurso psicológico que, si está disponible para el individuo, atenúa los efectos negativos de los eventos estresantes de la vida. Axelsson y Ejlertsson (2002) han añadido que entre los desempleados, el apoyo social percibido por parte de los padres parece ejercer mayor influencia que el resto. En lo referente a los jóvenes en situación de desempleo, Lorenzini y Giugni (2011) afirman que el apoyo social percibido en todas sus manifestaciones juega un papel crucial en la reducción del estrés psicológico asociado al desempleo. Varios autores (Chakrapani, 1996; Hammer, 2000; Hendry y Raymond, 1986) han añadido, además, que, entre los desempleados jóvenes, el apoyo social percibido que parece tener un mayor efecto paliativo en el estrés psicológico es el proveniente de los padres. Asimismo, en el estudio realizado por Bjarnason y Sigurdardottir (2003) en jóvenes de entre 18 y 24 años en situación de desempleo, se encontró que el apoyo proveniente de los padres tenía un efecto negativo en el estrés psicológico de jóvenes desempleados. A pesar de que muchas veces se hable de apoyo social en la literatura, se ha prestado atención especial a la percepción del apoyo social que tienen los individuos (Russell, Holmstrom y Clare, 2015). Se ha comprobado que el apoyo social percibido es un mejor predictor de la salud mental de los individuos que el apoyo social per se. Por ejemplo, McDowell y Serovich (2007) realizaron un estudio para comparar los efectos del apoyo social y los efectos del apoyo social percibido en la salud mental de personas $\mathrm{VIH}$-positivas y encontraron que, como norma general, el apoyo social percibido era significativamente más predictivo de la salud mental que el apoyo social per se. Así pues, parece ser que tiene mayor importancia la cantidad de apoyo social que el individuo percibe que tiene a su disposición, que la que en realidad, desde mediciones más "objetivas", tenga. Por su parte, a pesar de haberse descrito varios tipos de apoyo social (emocional, de compañía, informacional e instrumental), éstos se muestran tan correlacionados que se explican mejor mediante un único factor (Thoits, 1995). Aunque la mayor parte de la evidencia muestra que el apoyo social percibido se relaciona positivamente con la salud mental, hay también algo de evidencia al respecto de los límites de esta relación. Hay evidencia que muestra que una cantidad desmesurada de apoyo social puede no solo dejar de ser beneficioso, sino generar estrés en los individuos (Robinson y Tian, 2009). Así, la eficacia protectora del apoyo social sobre la salud mental, se dará sólo en aquellas situaciones en que la cantidad de apoyo social necesitado coincida con la cantidad proporcionada al individuo, al menos en su percepción (Russell, Holmstrom y Clare, 2015).

En cuanto a la resiliencia, ésta es la capacidad de adaptarse exitosamente a las situaciones a pesar de circunstancias desafiantes o que suponen una amenaza para el individuo (Masten, Best y 
Garmezy, 1990). En el contexto del desempleo, la resiliencia se entiende como la capacidad del individuo para hacer frente de forma exitosa a la adversidad personal y socioeconómica que supone el desempleo. Demostraciones de que una persona en situación de desempleo tiene un carácter resiliente sería que dicho individuo se esforzara por mantener un estado de ánimo positivo y llevara a cabo una búsqueda activa de empleo (Moorhouse y Caltabiano, 2007). Por tanto, el carácter resiliente de los jóvenes desempleados deberá actuar como un factor protector en cuanto a los efectos negativos que el desempleo posee para la salud mental.

Finalmente, las estrategias de afrontamiento que emplee el individuo también actuarán potencialmente como un factor protector para la salud mental de éste. Las estrategias de afrontamiento o coping comprenden el uso de recursos emocionales y cognitivos con el fin de manejar las situaciones que generan estrés (Lazarus y Folkman, 1984). Así, las estrategias de afrontamiento reflejan los comportamientos adoptados por las personas para alcanzar sus objetivos. Se han observado dos estrategias de afrontamiento exhibidas con frecuencia por los individuos en situación de desempleo: las estrategias de afrontamiento centradas en el problema y las estrategias de afrontamiento centradas en las emociones (Andersen, 2011; Lazarus y Folkman, 1984). Asimismo, Andersen (2011) encontró un comportamiento diferencial respecto al reempleo en función del tipo de estrategias de afrontamiento que predominaban; las estrategias centradas en el problema tenían un efecto positivo en el reempleo mientras que las estrategias centradas en el distanciamiento tenían un efecto negativo. Los individuos que exhiben estrategias enfocadas al problema suelen intentar tratar con él de forma directa, interactuando con el entorno. Por ejemplo, intentando encontrar otro trabajo. Las estrategias enfocadas a las emociones suelen exhibirse en individuos que regulan sus emociones negativas alterando sus procesos internos en lugar del entorno externo. Por ejemplo, negándose a sí mismos que se encuentran en situación de desempleo o que ésta pueda durar mucho (Langens y Mose, 2006). Con el fin de operacionalizar los dos tipos de afrontamiento en el contexto del desempleo, se han utilizado la búsqueda activa de empleo y el distanciamiento para medir las estrategias enfocadas a los problemas y a las emociones, respectivamente (Gowan, Riordan y Gatewood, 1999). La búsqueda activa de empleo consiste en que el individuo dedique su tiempo y energía en actividades relacionadas con la búsqueda de empleo. El distanciamiento se refiere al esfuerzo cognitivo por parte del individuo para escapar a la situación de desempleo, intentando no pensar en ello, pensando que el tiempo solucionará la situación, etc. (Lin y Leung, 2010). Según Lazarus y Folkman (1984), las estrategias de afrontamiento tienen dos funciones principales: tratar con el problema y regular el estrés emocional que lo acompaña. Kinicki et al. (2000), en su estudio meta-analítico, encontraron que, en general, son las estrategias centradas en el problema y no las centradas en las emociones, es decir, la búsqueda activa de empleo, lo que aumenta las posibilidades de encontrar un nuevo trabajo y, por tanto, suplen la primera función. En cambio, numerosos autores (Julkunen, 2001; Lai y Chan, 2002; Lin y Leung, 2010) afirman que son el distanciamiento o estrategias enfocadas en las emociones las que actúan como atenuante de los efectos negativos que tiene el desempleo sobre la salud mental. Por ejemplo, en un estudio longitudinal realizado por Lin y Leung (2010) en 1070 desempleados pertenecientes a un programa de asistencia para desempleados, se encontró que las estrategias de distanciamiento tenían un efecto positivo en diferido sobre la salud mental. Esto va en línea con Lazarus y Folkman (1984), según los cuales la confrontación prolongada con una situación estresante puede llevar al agotamiento si se emplean estrategias centradas en el problema sin éxito durante un período largo de tiempo. A esto se le suma evidencia que indica que las experiencias estresantes de corta duración están asociadas con estrategias de enfrentamiento centradas en el problema, mientras que las experiencias de larga duración se relacionan con estrategias de afrontamiento enfocadas en las emociones (Harnish, Aseltine y Gore, 2000).

En Angola, la situación de desempleo joven y sus posibles implicaciones en la salud mental son acuciantes, puesto que se estima un porcentaje aproximado del $26 \%$ de desempleados jóvenes (Ministry of Education, 2014). Este estudio tiene como objetivo estudiar las implicaciones que tiene el desempleo en la salud mental del sector joven de la población angoleña. Para ello, se ha especificado un Modelo de Ecuaciones Estructurales (MEE) completo con el objetivo de estudiar los valores predictivos del apoyo social percibido (de familia, amigos y otros), la resiliencia y dos estrategias de afrontamiento comúnmente empleadas en este contexto (búsqueda de empleo y distanciamiento) sobre tres aspectos de la salud mental: disforia, funcionamiento social y pérdida de confianza. Las hipótesis del estudio incluyen:

- Los jóvenes que perciban apoyo social de familia, amigos u otros presentarán un mejor funcionamiento social y menor disforia y pérdida de confianza que aquellos que no lo perciban.

- La resiliencia minimiza el desarrollo de disforia y pérdida de confianza, y aumenta el funcionamiento social. 
- Todos los tipos de coping, tanto la búsqueda activa de empleo como el distanciamiento, presentan efectos positivos sobre la salud mental: menor disforia y pérdida de confianza y mejor funcionamiento social.

\section{Muestra y procedimiento}

\section{Método}

La muestra la componen un total de 283 jóvenes angoleños desempleados. De ellos el $59.4 \%$ son hombres y el restante $40.6 \%$ mujeres, con edades comprendidas entre los 18 y los 30 años (Media= 23.3 años y DT=3.12). Llevan, como promedio, unos 22.8 meses en desempleo ( $D T=18.07$ ). La mayoría de los jóvenes de la muestra vivían en un entorno urbano (68.9\%), frente a un $31.1 \%$ que vivían en entorno rural. Por provincias, el grueso de la muestra provenía de Luanda, la capital del país, con un $62.9 \%$ de la muestra, de Benguela eran un $33.2 \%$ y residían en Uige el restante $3.9 \%$. El $88.7 \%$ declara no disponer de casa propia, y sí la poseen el $11.3 \%$ restante. En cuanto a con quién viven, la mayoría todavía con los padres $(82.7 \%)$, solos viven el $12.4 \%$, y con amigos un $4.9 \%$. La mayoría de los datos fueron tomados en Los Centros de Empleo, aunque también se tomaron algunos en centros educativos y deportivos. Previo a la participación de los jóvenes, se solicitaron los permisos oportunos a las autoridades de esos centros. Todos los participantes fueron informados sobre la naturaleza del estudio, además de la garantía de la confidencialidad de los datos y el derecho a participar libremente o rechazar la invitación. La respuesta de los jóvenes fue satisfactoria en cuanto a colaboración con la investigación. EI tiempo aproximado de aplicación de los cuestionarios fue inferior a 30 minutos. Al elegir la muestra se tuvo en cuenta que una gran parte de la población desempleada de Angola se encuentra en la capital, Luanda. Por otro lado, la provincia de Benguela, que se encuentra en la región centro-sur de Angola, es una de las más solicitadas para la búsqueda de empleo, dado su nivel de desarrollo. Finalmente, Uíge fue elegida por ser una de las provincias del norte de Angola con un buen desarrollo agrícola y el surgimiento de algunas empresas capaces de proporcionar empleo.

\section{Instrumentos de medida}

- Sociodemográficos. Se incluyen variables tales como la edad, el sexo (género), la escolaridad, el lugar de residencia (zona rural, zona urbana), la provincia, el número de hijos, el vínculo con alguna asociación, la práctica deportiva, el vínculo laboral, el conocimiento del centro de empleo, la búsqueda de trabajo, la preparación para trabajar, las formas de supervivencia, etc.

- Apoyo. Para evaluar el apoyo social se utilizó la Multidimensional Scale of Perceived Social Support (MSPSS, Zimet, Dahlem, Zimet y Farley, 1988), en versión de Edwards (2004). Está compuesta por doce ítems y evalúa tres dimensiones: Apoyo de la Familia, Apoyo de los Amigos, y Apoyo de los Otros Significativos. Ejemplos de ítems de esta escala son los siguientes: "Mi familia es una verdadera fuente de confort para mí", "Tengo amigos con quienes puedo compartir mis alegrías y tristezas", "Hay una persona especial que está cerca de mí cuando necesito de ella". Los jóvenes debían responder según las siguientes alternativas: desde (1) Totalmente en desacuerdo, hasta (5) Totalmente de acuerdo. La consistencia interna, medida mediante Composite Reliability Indexes (CRIs) fue de .77, .79 y .75 para apoyo familiar, de amigos y de otros respectivamente.

- Resiliencia. La escala de resiliencia utilizada fue la Brief Resilience Scale-BRS, de Smith et al. (2008). Es una escala unidimensional y está compuesta por seis ítems (tres redactados en sentido positivo y tres en negativo) (ejemplos: "Tengo tendencia a desistir rápidamente ante las dificultades", "No tardo mucho tiempo en recuperarme de un acontecimiento estresante"). Según sus autores, esta escala agrupa los seis ítems en un solo factor. Cada ítem permitía responder con una de las siguientes alternativas: desde (1) Totalmente en desacuerdo, hasta (5) Totalmente de acuerdo. Se eliminaron dos ítems, 1 y 3 , por un muy deficiente funcionamiento psicométrico. En concreto, las saturaciones factoriales en un factorial confirmatorio previo estaban por debajo de .25 en ambos casos y las correlaciones ítem-total corregidas ni siquiera alcanzaban estos valores. EI CRI para los restantes ítems valió .61.

- Estrategias de afrontamiento o coping. Para evaluar las estrategias de coping frente al desempleo, se utilizó la Coping With Job Loss Scale (CWJLS), versión de Lai y Wong (1998). La versión inglesa original de este instrumento fue desarrollada por Latack (1986) para medir el coping ante el estrés laboral en general, aunque también contenía aspectos de coping ante otras situaciones fuera del trabajo. Los 39 ítems que formaban la escala original fueron adaptados para medir específicamente el coping ante la pérdida del trabajo (Kinicki y Latack, 1990). Sucesivos tratamientos de esta escala la fueron reduciendo hasta quedar formada por los 11 ítems 
que ahora la componen (Lai y Wong, 1998). Aunque originariamente la CWJLS contaba con tres factores, la factorización desarrollada por Lai y Wong a partir de una muestra de personas desempleadas aportó únicamente dos factores: (1) Búsqueda proactiva de empleo, formado por cinco ítems que miden el grado de compromiso en la búsqueda activa de empleo (ejemplo: Me esfuerzo para encontrar un nuevo trabajo); y (2) Distanciamiento de la pérdida del empleo, compuesto por seis ítems que evalúan las estrategias de evasión y re-interpretación cognitiva (ejemplo: Me recuerdo a mí mismo que no tener trabajo no es el fin del mundo). Para completar la escala, los participantes debían responder a la siguiente pregunta: ¿Con qué frecuencia utiliza las siguientes estrategias para superar el desempleo? Por favor, responda indicando desde (1) Casi nunca, hasta (5) Casi siempre. La consistencia interna, medida mediante CRI fue de .79 y .56 para búsqueda activa de empleo y distanciamiento, respectivamente.

- Salud mental. Para medir la salud mental, se utilizó el General Health Questionnaire (GHQ, Goldberg y Williams, 1988). El GHQ es una medida destinada a evaluar el bienestar mental a partir del funcionamiento saludable normal y la aparición de nuevos síntomas de angustia y preocupación. Fue diseñado originariamente con 60 ítems y sucesivamente se han ido desarrollando versiones más cortas, siendo la más utilizada últimamente la versión de 12 ítems (GHQ12). Argile (1989) argumentaba que el GHQ es el indicador más fiable de la angustia psicológica (psychological distress), considerado inicialmente como un indicador del estado de salud mental. La puntuación general de la salud mental se deriva de la suma de las puntuaciones de los 12 ítems. Los jóvenes angoleños desempleados debían contestar a la pregunta: ¿Con qué frecuencia ha experimentado, a lo largo de las últimas semanas, las condiciones descritas en las siguientes expresiones? Por favor, responda indicando desde (1) Raramente hasta (5) Con mucha frecuencia. Algunos ejemplos de ítems son: "Fue capaz de concentrarse en lo que estaba haciendo?", "Perdió el sueño por causa de las preocupaciones?". En recientes investigaciones (Tomás, Gutiérrez y Sancho, 2017) se ha encontrado que mide tres dimensiones de disforia, funcionamiento social y pérdida de confianza. La consistencia interna (CRI) fue de $.67, .63$ y .57 para disforia, funcionamiento social y pérdida de confianza, respectivamente.

Todos estos cuestionarios fueron traducidos al portugués, lengua oficial en Angola, y que es la lengua vehicular y no sólo académica de todos los jóvenes angoleños. Los cuestionarios fueron sometidos al procedimiento habitual de back-translation. Todos los cuestionarios medían en escala de 1 (mínimo) a 5 (máximo).

\section{Análisis estadístico}

Se han realizado análisis descriptivos básicos en el SPSS 22. Adicionalmente, los análisis estadísticos centrales consisten en la puesta a prueba de un modelo de ecuaciones estructurales con variables latentes para predecir la salud mental de los jóvenes desempleados. El modelo estructural se ha estimado y puesto a prueba en el paquete estadístico EQS 5.7 mediante estimación máximo verosímil con correcciones robustas dado el alejamiento del supuesto de normalidad de las variables. Como recomienda la literatura (Kline, 2011; Tanaka, 1993) se calcularon varios estadísticos e índices de ajuste: el estadístico chi-cuadrado $\left(\mathrm{X}^{2}\right)$, el Comparative Fit Index (CFI), Root Mean Square Error of Approximation (RMSEA) y Standardized Root Mean Residual (SRMR). Generalmente, se asume un buen ajuste cuando el CFI es igual o mayor a .90, aunque es más satisfactorio un CFI de .95 o superior, junto con medidas del tamaño del error como son el RMSEA o el SRMR menores a .08 e idealmente menores de .05 (Hu y Bentler, 1999). La fiabilidad de las variables latentes en el modelo se calculó mediante los correspondientes CRI (Composite Reliability Indexes; Raykov, 2004). El tamaño muestral no es excesivamente elevado y el número de indicadores, si se tomaban todos los ítems de cada una de las escalas, demasiado grande, dando lugar a una ratio desaconsejablemente pequeña de sujetos por parámetro a estimar. Por ello se optó por una alternativa analítica, realizar un agrupamiento de los ítems o "item parceling" para cada una de las variables latentes del modelo. En el procedimiento de "item parceling" los ítems se agrupan para formar paquetes de información tratándolos como variables observables. El procedimiento de parceling ofrece parsimonia con respecto al tamaño muestral y se ha recomendado en el caso de modelos de ecuaciones estructurales con variables latentes complejas, como el que se presenta (Little, Rhemtulla, Gibson y Schoemann, 2013). Esto es, cuando se manejan escalas largas o muestras relativamente pequeñas (Yang, Nay y Hoyle 2010). Estudios de simulación han probado la eficiencia del agrupamiento de ítems (Nasser y Wisenbaker, 2003). Cuando se cumple la unidimensionalidad prácticamente cualquier agrupamiento en paquetes de los ítems de una escala produce buenos resultados (Bandalos y Finney, 2001). En este caso se han agrupado los pares adyacentes de ítems. 


\section{Resultados}

Los estadísticos descriptivos, media y desviación típica, junto con los índices de asimetría y curtosis de todos los indicadores del modelo se ofrecen en la Tabla 1.

\section{Tabla 1}

Medias, desviaciones típicas, asimetría y curtosis para cada uno de los indicadores del modelo. Nota: Para el indicador ayuda para sobrevivir, dicotómico, solamente se presenta la media que coincide con la proporción de jóvenes que no necesita ayuda para sobrevivir.

\begin{tabular}{|c|c|c|c|c|}
\hline Indicador & $\mathrm{M}$ & DT & AS & $\mathrm{CU}$ \\
\hline Ayuda para sobrevivir $(0=$ sí, $1=$ no) & 0.27 & & & \\
\hline Apoyo familiar Parcel 1 & 3.80 & 1.12 & -1.034 & 0.468 \\
\hline Apoyo familiar Parcel 2 & 3.75 & 1.08 & -0.746 & -0.032 \\
\hline Apoyo amigos Parcel 1 & 3.31 & 1.13 & -0.540 & -0.418 \\
\hline Apoyo amigos Parcel 2 & 3.63 & 1.00 & -0.610 & -0.003 \\
\hline Apoyo otros Parcel 1 & 3.89 & 1.02 & -1.150 & 1.024 \\
\hline Apoyo otros Parcel 2 & 4.05 & 0.98 & -1.165 & 0.857 \\
\hline Resiliencia Parcel 1 & 2.97 & 0.97 & 0.007 & -0.472 \\
\hline Resiliencia Parcel 2 & 3.12 & 0.96 & -0.073 & -0.355 \\
\hline Búsqueda activa Parcel 1 & 3.80 & 1.25 & -0.084 & -1.455 \\
\hline Búsqueda activa Parcel 2 & 3.98 & 1.20 & -0.319 & -1.281 \\
\hline Búsqueda activa Parcel 3 & 4.07 & 1.26 & -0.591 & -1.262 \\
\hline Distanciamiento Parcel 1 & 3.24 & 1.27 & 0.017 & -0.999 \\
\hline Distanciamiento Parcel 2 & 2.97 & 1.21 & 0.127 & -0.908 \\
\hline Distanciamiento Parcel 3 & 3.63 & 1.12 & -0.335 & -0.837 \\
\hline Disphoria Parcel 1 & 2.92 & 1.23 & 0.007 & -0.941 \\
\hline Disphoria Parcel 2 & 2.77 & 1.29 & 0.172 & -0.945 \\
\hline Funcionamiento social Parcel 1 & 3.61 & 1.15 & -0.598 & -0.346 \\
\hline Funcionamiento social Parcel 2 & 3.58 & 1.16 & -0.606 & -0.126 \\
\hline Funcionamiento social Parcel 3 & 3.65 & 1.13 & -0.589 & -0.197 \\
\hline Pérdida de confianza Parcel 1 & 2.31 & 1.50 & 0.778 & -0.879 \\
\hline Pérdida de confianza Parcel 2 & 2.34 & 1.54 & 0.729 & -1.012 \\
\hline
\end{tabular}

El modelo que se puso a prueba era un modelo predictivo mediante variables latentes de las tres dimensiones de salud mental consideradas, a saber, la disforia, el funcionamiento social y la pérdida de confianza. Las dimensiones predictivas (predictores) considerados fueron el apoyo social (tres factores, apoyo de los familiares, de los amigos y de otros significativos), la resiliencia y las estrategias de coping ante la situación de desempleo (búsqueda activa de empleo y distanciamiento). Adicionalmente se añadió como predictor un indicador dicotómico (0/1) donde el 1 indica que el joven, pese a estar desempleado, se mantiene por sí mismo. El objetivo era controlar estadísticamente por los efectos de ser económicamente (relativamente) suficiente, más allá de la situación de desempleo. Adicionalmente se hipotetizó en el modelo que las dimensiones de salud mental mostrarían correlaciones entre sí, así como las dimensiones exógenas, también se plantearon como ortogonales.

Este modelo resultó en un ajuste adecuado, pese a que la prueba de chi-cuadrado ha resultado significativa, ya que los estadísticos e índices de ajuste fueron adecuados: $\chi_{167}^{2}=256.47, p<.001$; CFI $=.925 ;$ SRMR $=.051 ;$ y RMSEA = .046 [.034 - .057]. Los predictores se encontraron correlacionados significativamente entre ellos en su mayor parte. Estos valores de correlación, que no se muestran en la Figura 1, por simplicidad, se ofrecen como matriz de correlaciones en la Tabla 2. Los parámetros de interés del modelo estructural se presentan en la Figura 1. Por lo que hace a los efectos sobre la disforia, solamente el factor de resiliencia presentaba un efecto estadísticamente significativo y negativo sobre la disforia. Es decir, a mayor resiliencia del joven, menor grado de disforia. El porcentaje de varianza de disforia explicada fue del $34.7 \%$ por la resiliencia. Por lo que hace al buen funcionamiento social, éste se ve favorecido de forma estadísticamente significativa por un adecuado apoyo familiar y por distanciarse de la situación estresante. Esto es, el desempleo. Así, el funcionamiento social fue predicho en un $40.5 \%$ por estas dimensiones, finalmente, también dos factores afectaron negativamente, si bien uno de ellos sólo marginalmente $(p<.1)$, al factor de pérdida de confianza: la resiliencia de manera importante y el apoyo de amigos. En ambos casos, si se aumenta la resiliencia o el apoyo de amigos se baja la pérdida de confianza del desempleado. La varianza explicada de la pérdida de confianza resultó ser en un $22.2 \%$. En cuanto a las correlaciones hipotetizadas entre las dimensiones de salud mental, la única significativa $(p<.05)$, es la positiva entre la disforia y la pérdida de confianza. 
Tabla 2

Correlaciones entre las variables predictivas.

\begin{tabular}{|c|c|c|c|c|c|c|}
\hline & Ayuda & Apoyo familia & Apoyo amigos & Apoyo otros & Resiliencia & Búsqueda activa \\
\hline \multicolumn{7}{|l|}{ Ayuda } \\
\hline Apoyo familia & $-.128^{*}$ & & & & & \\
\hline Apoyo amigos & -.087 & $.405^{*}$ & & & & \\
\hline Apoyo otros & -.100 & $.534^{*}$ & $.397^{*}$ & & & \\
\hline Resiliencia & .099 & .049 & $.141^{*}$ & $.146^{*}$ & & \\
\hline Búsqueda activa & .089 & $.210^{*}$ & .117 & $.262^{*}$ & $.127^{*}$ & \\
\hline Distanciamiento & .021 & $.184^{*}$ & $.125^{*}$ & $.321^{*}$ & $.125^{*}$ & $.327^{*}$ \\
\hline
\end{tabular}

Nota: ${ }^{*}=p<.05$

\section{Figura 1}

Modelo final para predecir salud mental. Notas: Todas las relaciones estadísticamente significativas $(p<.05)$ salvo una $(m s=$ marginalmente significativa $p<.1)$. Los coeficientes estructurales que se muestran, por simplicidad, son solamente los estadísticamente (o marginalmente) significativos. De la misma forma, no se muestran las correlaciones entre las variables independientes, que se mostrarán en tabla.

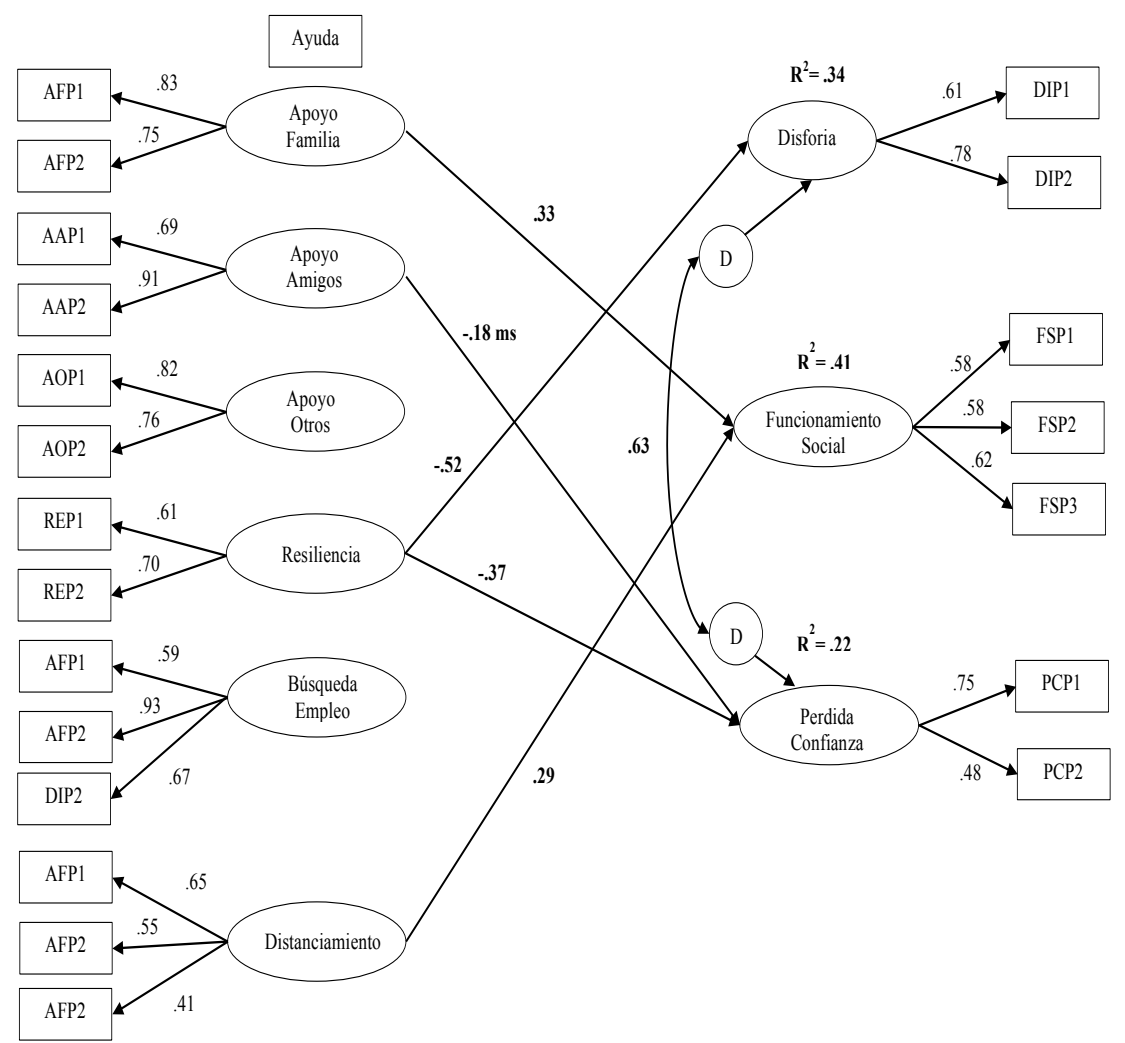

\section{Conclusiones y discusión}

El objetivo de la presente investigación consistía en valorar la capacidad predictiva de diversos factores sobre la salud mental de jóvenes desempleados angoleños. Todo ello en un contexto multivariado que permitiera discernir qué factores pueden ser protectores frente a la previsiblemente empeorada salud mental motivada por el desempleo. Se han considerado factores económicos (capacidad de mantenerse aun estando en situación de desempleo), sociales (apoyo social), personales (resiliencia), así como el uso de estrategias para afrontar el problema (búsqueda activa de empleo y distanciamento). $Y$ se ha evaluado su impacto sobre una medida ampliamente empleada para cribar problemas mentales menores subclínicos, como es el General Health Questionnaire y sus tres dimensiones de disforia, funcionamiento social y la pérdida de confianza. Los resultados en general muestran que la salud mental se ve fuertemente impactada tanto por factores personales como sociales. Entre los factores personales el más relevante resultó la resiliencia, seguido de la capacidad de distanciarse de la situación de desempleo. De entre los sociales el apoyo de la familia fue el más predictivo.

Una primera conclusión relevante es que el modelo estructural planteado presentó un muy buen ajuste. Encadenado con este resultado se puede también apreciar la capacidad predictiva del modelo, 
pues en el peor de los casos los factores predicen un $22 \%$ de la pérdida de confianza, pero hasta un $41 \%$ del funcionamiento social. Otro resultado relevante es que los factores predictores se encuentran altamente relacionados entre sí. Es decir, más allá de que sean algunos factores concretos los que predigan significativamente en mayor o menor medida la salud mental en sus tres dimensiones, todos los predictores se encuentran relacionados entre sí y de alguna forma, se catalizan.

Los resultados concretos de predicción obtenidos reflejan, pues, la relevancia de los factores considerados en la literatura. Según con lo hipotetizado, un resultado relevante es la importancia del apoyo social percibido por el individuo y su consecuente efecto positivo en la salud mental. En concreto, y según lo indicado en la literatura, el apoyo social proveniente de los padres resultó ser el más importante y el único que mostró un efecto significativo como predictor de la salud mental, concretamente, de un mejor funcionamiento social (Axelsson y Ejlertsson, 2002; Chakrapani, 1996; Hammer, 2000; Hendry y Raymond, 1986). Esto puede deberse a que una de las principales dificultades para los jóvenes en situación de desempleo es la de carácter económico, y se ha visto que la familia es, en estos casos, el principal proveedor de apoyo (Giuntoli, South, Kisella y Karban, 2011). Por su parte, el apoyo social procedente de los amigos y de otras fuentes no muestra poder predictor sobre ninguna de las dimensiones de la salud mental.

En lo que hace a la resiliencia, los resultados muestran su papel central en la predicción de la salud mental. La resiliencia disminuiría la disforia, así como sobre la pérdida de confianza, lo cual va a favor de la evidencia establecida en torno al papel de la resiliencia en la salud mental (Moorhouse y Caltabiano, 2007). El efecto de la resiliencia es el más importante, y lo es en los aspectos más emocionales de la salud mental, el estado de disforia y la pérdida de confianza. Esto no son excesivas buenas noticias si se considera la naturaleza relativamente estable de ésta, pero en la vertiente positiva, señala a la dirección de favorecer la conducta resiliente de los jóvenes como vía de mejora de su salud mental. Adicionalmente, los propios Moorhouse y Caltabiano (2007) señalaban que era esperable que la resiliencia correlacionara positivamente con la búsqueda activa de empleo. Esto ha sido así en nuestros datos, si bien de manera modesta. Más interesante es ver que esta misma asociación positiva, y prácticamente de la misma cuantía, se ha dado con el distanciamiento. Esto indica que los resilientes tienden a aumentar el uso de estrategias de coping (afrontamiento), sean éstas del tipo que sean, activas o de evitación.

Por último, entre las estrategias de afrontamiento, la única que ejerce un efecto predictor sobre la salud mental, concretamente el funcionamiento social, es el distanciamiento de los individuos hacia la situación de desempleo, que afecta positivamente. Este resultado va en línea con la idea de que las estrategias enfocadas a las emociones ayudan al individuo a distanciarse de la situación de desempleo y así escapar de los efectos negativos que ésta tiene sobre la salud mental (Julkunen, 2001; Lai y Chan, 2002; Lin y Leung, 2010). También de acuerdo con lo establecido por Lin y Leung (2010), la búsqueda activa de empleo no mostró efectos en ninguna de las dimensiones de salud mental. La no relación de la búsqueda activa de empleo no era lo esperado de acuerdo a la literatura ya que Kinicki et al. (2000) encontraron que las estrategias centradas en el problema y no las centradas en las emociones, es decir, la búsqueda activa de empleo, las que aumentan las posibilidades de encontrar un nuevo trabajo y de acuerdo a eso esperábamos un mejor funcionamiento y salud mental en los que las emplean. Sin embargo, otros autores ponen de manifiesto que el distanciamiento o estrategias enfocadas en las emociones, en situaciones de desempleo alargadas en el tiempo, actúan como atenuante de los efectos negativos que tiene el desempleo sobre la salud mental (Julkunen, 2001; Lai y Chan, 2002; Lin y Leung, 2010). Es en este sentido en que deben interpretarse los resultados hallados. Posiblemente los que buscan más activamente empleo ya estén empleados, o al menos están menos representados en la muestra. Diseños longitudinales, en los que se pueda seguir a los desempleados un tiempo suficiente para observar cambios en su empleabilidad ayudarán a discernir mejor las razones del resultado encontrado.

Cabe destacar las implicaciones derivadas de este estudio. En primer lugar, el hecho de que tan solo el apoyo social percibido de los padres muestre un efecto predictor significativo indica que son éstos los que proveen a los jóvenes con mayor cantidad y calidad de apoyo, y por tanto es conveniente promover esta relación en poblaciones donde se encuentra un alto porcentaje de jóvenes desempleados. En segundo lugar, la resiliencia juega un papel muy relevante. En tercer lugar, parece que aquellos jóvenes cuya situación se prolonga en el tiempo, optan por tomar distancia cognitiva de dicha situación, lo que mejora su salud mental de acuerdo a los resultados, pero desde el punto de vista práctico sabemos (Kinicki et al., 2000) que es la búsqueda activa de empleo la que aumenta las probabilidades de encontrarlo. Como ambas estrategias se muestran correlacionadas positivamente en nuestros resultados, parece ser que existe una alta probabilidad de emplear ambas estrategias conjuntamente. 
Mientras que el distanciamiento actuaría frente al deterioro de la salud mental, la búsqueda activa de empleo no se muestra efectiva para eso, pero sí para encontrar efectivamente empleo, por lo que las instituciones sociales necesitan encontrar modos de promover ambos tipos de coping entre los jóvenes.

Respecto a las limitaciones del estudio, las principales vienen de su carácter transversal y de la muestra incidental ya que imposibilita estudiar el desarrollo en el tiempo de la salud mental de los sujetos. El diseño transversal hace difícil valorar efectos causales, por lo que las conclusiones se mantienen al nivel puramente predictivo. La muestra incidental, aunque permite ver si las relaciones hipotetizadas, que se esperan de carácter general a cualquier población joven desempleada, se dan en la muestra, sin embargo por su no representatividad hace difícil generalizar los resultados exactamente en términos estadísticos a la población de jóvenes angoleños. Esto podría implicar un mayor conocimiento sobre los efectos del distanciamiento y de la búsqueda activa de empleo en el período de tiempo que abarque el desempleo. Por último, el presente estudio añade información a la literatura del desempleo, especialmente porque se ha llevado a cabo en una población, jóvenes desempleados angoleños, que hasta la fecha no había sido estudiada.

Los resultados aquí planteados, aunque de carácter transversal y con sus limitaciones, ofrecen ideas e implicaciones para la intervención. Un primer resultado relevante es la importancia relativa que aporta la resiliencia, que se presenta como el predictor más relevante de la salud mental. Aunque podría aducirse que esto dificulta hablar en términos de intervención, pues muchos autores asimilan la resiliencia a un rasgo de personalidad, lo cierto es que hay evidencia acumulada de que puede intervenirse para modificar los niveles de resistencia (Cornum, Matthews y Seligman, 2011; Karatsoreos y McEwan, 2011). Una segunda implicación de los resultados es que el apoyo proveniente de la familia parece ser más protector que el de los amigos en esa etapa vital y para personas desempleadas. Así pues, debiera ser el que habría que fomentar. Esto posiblemente se deba a que en Angola los padres están, probablemente, en mejor disposición de dar un apoyo, no solo afectivo sino instrumental. Y, finalmente, los resultados apuntan a que el distanciamiento puede ser efectivo en la mejora de la salud mental, y en este contexto puede que más que la búsqueda activa de empleo. No obstante, este resultado habría que ponerlo en cuarentena ante la falta de evidencia longitudinal, pues podría ser que aquellos con tendencia a un coping focalizado en el problema, (búsqueda de empleo) sean más exitosos y salgan del grupo de desempleados, con lo que se produciría un sesgo.

\section{Referencias}

1. Amundson, N. (1994). Negotiating Identity During Unemployment. Journal of Employment Counselling, 31(3), 98-104. https://doi.org/10.1002/j.2161-1920.1994.tb00178.x

2. Amundson, N. \& Borgen, W. (1987). Coping with Unemployment: What Helps and What Hinders. Journal of Employment Counselling, 24(3), 97-106. https://doi.org/10.1002/j.2161-1920.1987. tb00222.x

3. Andersen, S. (2011). Exiting unemployment: How do program effects depend on individual coping strategies? Journal of Economic Psychology, 32(2), 248-258. https://doi.org/10.1016/j. joep.2010.01.013

4. Argyle, M. (1989). The psychology of happiness. London: Routledge.

5. Axelsson, L. \& Ejlertsson, G. (2002). Self-reported health, self-esteem and social support among young unemployed people: a population-based study. International Journal of Social Welfare, 11(2), 111-119. https://doi.org/10.1111/1468-2397.00205

6. Bandalos, D. L., \& Finney, S. J. (2001). Item parceling issues in structural equation modeling. In G. A. Marcoulides \& R. E. Schumacker (Eds.), New developments and techniques in structural equation modeling (pp. 269-296). Mahwah, NJ: Lawrence Erlbaum Associates, Inc.

7. Bjarnason, T. \& Sigurdardottir, T. (2003). Psychological distress during unemployment and beyond: social support and material deprivation among youth in six northern European countries. Social Science \& Medicine, 56(5), 973-985. https://doi.org/10.1016/S0277-9536(02)00109-0

8. Björklund, A. \& Eriksson, T. (1998). Unemployment and mental health: evidence from research in the Nordic countries. Scandinavian Journal of Social Welfare, 7(3), 219-235. https://doi. org/10.1111/j.1468-2397.1998.tb00286.x

9. $\quad$ Chakrapani, C. (1996). Unemployment as a stressor. Indian Journal of Social Work, 57(4), 578590.

10. Cobb, S. (1976). Social support as a moderator of life stress. Psychosomatic Medicine, 38(5), 300-314. https://doi.org/10.1097/00006842-197609000-00003

11. Cornum, R., Matthews, M. E, \& Seligman, M. E. P. (2011). Comprehensive Soldier Fitness: Building resilience in a challenging institutional context. American Psychologist, 66, 4-9. https://doi. org/10.1037/a0021420 
12. Edwards, L.M. (2004). Measuring perceived social support in Mexican American youth: Psychometric properties of the Multidimensional Scale of Perceived Social Support. Hispanic Journal of Behavioral Sciences, 26(2), 187-194. https://doi.org/10.1177/0739986304264374

13. Feather, N. \& O'Brien, G. (1986). A longitudinal analysis of the effects of different patterns of employment and unemployment on school-leavers. British Journal of Psychology, 77(4), 459479. https://doi.org/10.1111/j.2044-8295.1986.tb02211.x

14. Giuntoli, G., South, J., Kisella, K. \& Karban, K. (2011). Mental health, resilience and the recession in Bradford. Project Report. Joseph Rowntree Foundation.

15. Goldberg, D. \& Williams, P. (1988). A user's guide to the General Health Questionnaire. NFER-Nelson, UK.

16. Gowan M. A., Riordan C. M., \& Gatewood R. D. (1999). Test of a model of coping with involuntary job loss following a company closing. Journal of Applied Psychology, 84(1), 75-86. https://doi. org/10.1037/0021-9010.84.1.75

17. Hammer, T. (2000). Mental health and social exclusion among unemployed youth in Scandinavia: A comparative study. International Journal of Social Welfare, 9(1), 53-63. https://doi. org/10.1111/1468-2397.00108

18. Harnish, J. D., Aseltine, R. H., \& Gore, S. (2000). Resolution of stressful experiences as an indicator of coping effectiveness in young adults: An event history analysis. Journal of Health and Social Behavior, 41(2), 121-136. https://doi.org/10.2307/2676301

19. Hendry, L. B., \& Raymond, M. J. (1986). Psycho-social aspects of youth unemployment: An interpretative model. Journal of Adolescence, 9(4), 355-366. https://doi.org/10.1016/S0140$1971(86) 80041-0$

20. Jin, R. L., Shah, C. P., \& Svoboda, T. J. (1995). The Impact of Unemployment on Health: A Review of the Evidence. Journal of Public Health Policy, 18(3), 275-301. https://doi.org/10.2307/3343311

21. Julkunen, I. (2001). Coping and mental well-being among unemployed youth: ANorthern European perspective. Journal of Youth Studies, 4(3), 261-278. https://doi.org/10.1080/13676260120075419

22. Karatsoreos, I. N., \& McEwen, B. S. (2011). Psichobiological allostasis: resistence, resilience and vulnerability. Trends in Cognitive Science, 15, 576-582. https://doi.org/10.1016/j.tics.2011.10.005

23. Kinicki, A.J. \& Latack, J.C. (1990). Explication of the construct of coping with job loss. Journal of Vocational Behavior, 36, 339-360. https://doi.org/10.1016/0001-8791(90)90036-2

24. Kinicki, A. J., Prussia, G. E., \& McKee-Ryan, F. M. (2000). A panel study of coping with involuntary job loss. Academy of Management Journal, 43 (1), 90-100. https://doi.org/10.2307/1556388

25. Kline, R. B. (2011). Principles and practice of structural equation modeling. New York: The Guilford Press.

26. Kroll, L. E. \& Lampert, T. (2011) Unemployment, social support and health problems - results of the GEDA study in Germany, 2009. Deutsches Ärzteblatt International, 108(4), 47-52.

27. Lai, J. C. L. \& Chan, R. K. H. (2002). The effects of job-search motives and coping on psychological health and re-employment: A study of unemployed Hong Kong Chinese. International Journal of Human Resource Management, 13(3), 465-483. https://doi.org/10.1080/09585190110111486

28. Lai, J.C.L. \& Wong, W.S. (1998). Optimism and coping with unemployment among Hong Kong Chinese women. Journal of Research in Personality, 32(4), 454-479. https://doi.org/10.1006/ irpe.1998.2232

29. Langens, T. \& Mose, E. (2006). Coping With Unemployment: Relationships Between Duration of Unemployment, Coping Styles, and Subjective Well-Being. Journal of Applied Biobehavioral Research, 11(3-4), 189-208. https://doi.org/10.1111/j.1751-9861.2007.00005.x

30. Latack, J.C. (1986). Coping with job stress: Measures and future directions for scale development. Journal of Applied Psychology, 71, 377-385. https://doi.org/10.1037/0021-9010.71.3.377

31. Lazarus, R. S. \& Folkman, S. (1984). Stress, appraisal, and coping. New York: Springer.

32. Lin, X. \& Leung, K. (2010). Differing effects of coping strategies on mental health during prolonged unemployment: A longitudinal analysis. Human Relations, 63(5), 637-665. https://doi. org/10.1177/0018726709342930

33. Little, T. D., Rhemtulla, M., Gibson, K., \& Schoemann, A. M. (2013). Why the items versus parcels controversy needn't be one. Psychological Methods, 18, 285-300. https://doi.org/10.1037/ a0033266

34. Lorenzini, J. \& Giugni, M. (2011). Youth Coping with Unemployment: the Role of Social Support. Revue Suisse de Travail Social, 11 (2), 80-99.

35. Masten, A. S., Best, K. M., \& Garmezy, N. (1990). Resilience and development: Contributions from the study of children who overcome adversity. Development and Psychopathology, 2(4), 425-444. https://doi.org/10.1017/S0954579400005812 
36. McDowell, T. \& Serovich, J. (2007). The effect of perceived and actual social support on the mental health of HIV-positive persons. AIDS Care, 19(10), 1223-1229. https://doi. org/10.1080/09540120701402830

37. McKee-Ryan, F., Song, Z., Wanberg, C., \& Kinicki, A. (2005). Psychological and Physical Well-Being During Unemployment: A Meta-Analytic Study. Journal of Applied Psychology, 90(1), 53-76. https://doi.org/10.1037/0021-9010.90.1.53

38. Ministry of Education, (2014). Country report on policies and mechanisms for integration into the workforce and job creation. Ministerio de Educacao: Luanda, Angola.

39. Moorhouse, A. \& Caltabiano, M. (2007). Resilience and unemployment: exploring risk and protective influences for the outcome variables of depression and assertive job searching. Journal of Employment Counselling, 44, 115-125. https://doi.org/10.1002/j.2161-1920.2007.tb00030.x

40. Nasser, F., \& Wisenbaker, J. (2003). A Monte Carlo Study Investigating the Impact of Item Parceling on Measures of Fit in Confirmatory Factor Analysis. Educational and Psychological Measurement, 63, 729-757. https://doi.org/10.1177/0013164403258228

41. Paul, K., \& Moser, K. (2009). Unemployment impairs mental health. Journal of Vocational Behavior, 74, 264-282. https://doi.org/10.1016/j.jvb.2009.01.001

42. Prussia G.E., Fugate M., \& Kinicki A. (2001) Explication of the coping goal construct: Implications for coping and reemployment. Journal of Applied Psychology, 86, 1179-1190. https://doi. org/10.1037/0021-9010.86.6.1179

43. Raykov, T. (2004). Behavioral scale reliability and measurement invariance evaluation using latent variable modeling. Behavioral Therapy, 35, 299-331. https://doi.org/10.1016/S00057894(04)80041-8

44. Robinson, J. D., \& Tian, Y. (2009). Cancer patients and the provision of informational social support. Health Communication, 24, 381-390. https://doi.org/10.1080/10410230903023261

45. Russell, J., Holmstrom, A. J., \& Clare, D. D. (2015). The Differential Impact of Social Support Types in Promoting New Entrant Job Search Self-Efficacy and Behavior, Communication Research Reports, 32, 170-179. https://doi.org/10.1080/08824096.2015.1016150

46. Schwarzer, R., Jerusalem, M., \& Hahn, A. (1994). Unemployment, social support and health complaints: A longitudinal study of stress in East German refugees. Journal of Community and Applied Social Psychology, 4, 31-45. https://doi.org/10.1002/casp.2450040109

47. Ślebarska, K., Moser, K., \& Gunnesch-Luca, G. (2009). Unemployment, social support, individual resources, and job search behavior. Journal of Employment Counselling, 46, 159-170. https://doi. org/10.1002/j.2161-1920.2009.tb00079.x

48. Smith, B.W., Dalen, J., Wiggins, K., Tooley, E., Christopher, P., \& Bernard, J. (2008). The Brief Resilience Scale: Assessing the ability to bounce back. International Journal of Behavioral Medicine, 15, 194-200. https://doi.org/10.1080/10705500802222972

49. Tanaka, J. S. (1993). Multifaceted conceptions of fit in structural equation models. In K. A. Bollen (Ed.), Testing structural equation models (pp. 10-39). Newbury Park, CA: Sage.

50. Thoits, P. A. (1995). Stress, Coping, and Social Support Processes: Where Are We? What Next? Journal of Health and Social Behavior, 35, 53-79. https://doi.org/10.2307/2626957

51. Tomás, J. M., Gutiérrez, M., \& Sancho, P. (2017). Factorial Validity of the General Health Questionnaire 12 in an Angolan Sample. European Journal of Psychological Assessment. https://doi. org/10.1027/1015-5759/a000278

52. Warr, P., Jackson, P., \& Banks, M. H. (1988). Unemployment and mental health: Some British studies. Journal of Social Issues, 44, 47-68. https://doi.org/10.1111/j.1540-4560.1988.tb02091.x

53. Winefield, A. (1997). Editorial: Introduction to the psychological effects of youth unemployment: international perspectives. Journal of Adolescence, 20, 237-241. https://doi.org/10.1006/ jado.1997.0082

54. Yang, C., Nay, S., \& Hoyle, R. H. (2010). Three Approaches to Using Lengthy Ordinal Scales in Structural Equation Models: Parceling, Latent Scoring, and Shortening Scales. Applied Psychological Measurement, 34, 122-142. https://doi.org/10.1177/0146621609338592

55. Zimet, G. D., Dahlem, N. W., Zimet, S. G., \& Farley, G. K. (1988). The Multidimensional Scale of Perceived Social Support. Journal of Personality Assessment, 52, 30-41. https://doi.org/10.1207/ s15327752jpa5201 2 